\title{
El liderazgo efectivo en los centros concertados de naturaleza cooperativa: Percepciones de sus docentes
}

Effective leadership in state-subsidised schools of cooperative nature: Perceptions of the teaching staff

\section{Volumen 18, Número 3 \\ Setiembre-Diciembre}

pp. 1-21

Este número se publica el 1 de setiembre de 2018

DOI: https://doi.org/10.15517/aie.v18i3.34195

\author{
Jesús López Belmonte \\ Arturo Fuentes Cabrera \\ Antonio José Moreno Guerrero
}

Revista indizada en REDALYC, SCIELO

Revista distribuida en las bases de datos:

LATINDEX, DOAJ, REDIB, IRESIE, $\underline{\text { CLASE}}$ DIALNET, SHERPA/ROMEO, QUALIS-CAPES, MIAR

Revista registrada en los directorios:

ULRICH'S $, \underline{R E D I E}, \underline{\text { RINACE}}, \underline{\text { OEI }}, \underline{\text { MAESTROTECA }}, \underline{\text { PREAL, }}$ 


\title{
El liderazgo efectivo en los centros concertados de naturaleza cooperativa: Percepciones de sus docentes
}

Effective leadership in state-subsidised schools of cooperative nature: Perceptions of the teaching staff

\author{
Jesús López Belmonte ${ }^{1}$ \\ Arturo Fuentes Cabrera² \\ Antonio José Moreno Guerrero ${ }^{3}$
}

\begin{abstract}
Resumen: El liderazgo es considerado un atributo de las personas que poseen ciertas habilidades para conseguir la dinamización del colectivo de una organización y modificar la actitud de los trabajadores con el propósito de aumentar la predisposición y el rendimiento de las personas. La finalidad de la presente investigación es conocer las percepciones docentes en torno al concepto de liderazgo en una cooperativa de enseñanza. El estudio desarrollado es de tipo descriptivo y correlacional, mediante un análisis desde un enfoque cuantitativo para toda la población de docentes que conforman las cooperativas de enseñanza de la Ciudad Autónoma de Ceuta, en España. La recopilación de datos se ha llevado a cabo mediante cuestionario, al cual se le ha aplicado la validez de constructo y la fiabilidad mediante Alfa de Cronbach. El análisis descriptivo describe cada una de las variables analizadas, mientras que el análisis correlativo se ha llevado a cabo a través la prueba de Chi-Cuadrado para saber si había relación entre las variables. Para conocer la fuerza de relación se ha aplicado la prueba Tau-b de Kendall. Los resultados muestran que no existe relación directa entre las variables género y liderazgo, y edad y liderazgo. Las conclusiones demuestran que el líder en educación debe incidir en todos los miembros que componen la comunidad educativa, con el fin de mejorar la calidad de los procesos de enseñanza-aprendizaje y fomentar un buen clima de relaciones sociales.
\end{abstract}

Palabras clave: liderazgo, cooperativas, educación.

\begin{abstract}
Leadership is considered an attribute of people who possess certain skills to achieve the dynamism of the collective of an organization and change the attitude of workers in order to increase the predisposition and performance of people. The purpose of this research is to know the perceptions of teachers about the concept of leadership in a teaching cooperative. The study developed is descriptive and correlational, through an analysis from a quantitative approach for the entire population of teachers who make up the teaching cooperatives of the Autonomous City of Ceuta, in Spain. The data collection was carried out by means of a questionnaire, to which the construct validity and reliability by means of Cronbach's Alpha have been applied. The descriptive analysis describes each of the analyzed variables, while the correlative analysis has been carried out through the Chisquare test to know if there was a relationship between the variables. To know the strength of the relationship, the Kendall Tau-b test was applied. The results show that there is no direct relationship between the variables gender and leadership, and age and leadership. The conclusions show that the leader in education must influence all the members of the educational community, in order to improve the quality of the teaching-learning processes and foster a good climate of social relations.
\end{abstract}

Key words: leadership, cooperatives, education.

1 Investigador de la Universidad de Granada, España; Grupo de Investigación AREA. Dirección electrónica: jesuslopezbelmonte@gmail.com

2 Docente de la Universidad de Granada, España; Grupo de Investigación AREA.

Dirección electrónica: arturofuentes@ugr.es

${ }^{3}$ Docente de la Universidad de Granada, España; Grupo de Investigación AREA.

Dirección electrónica: ajmoreno@ugr.es

Artículo recibido: 3 de marzo, 2018

Enviado a corrección: 25 de junio, 2018

Aprobado: 16 de julio, 2018

Volumen 18 Número 3 (Setiembre-Diciembre), ISSN 1409-4703 


\section{Introducción}

Vivimos en una sociedad caracterizada por la multitud de estímulos que condicionan la vida y las relaciones interpersonales, establecidas entre los miembros que conforman un determinado colectivo. Esto, sumado a la disparidad de diversos perfiles laborales dentro de una entidad, hace que el trabajo a desarrollar en una organización se encuentre condicionado por las características e intereses individuales de cada trabajador. Es en este punto donde adquiere gran relevancia la implantación y el desarrollo de un liderazgo eficaz dentro del grupo de trabajo. En este caso concreto, resulta primordial que todas las personas profesionales de la educación que fluctúan en un mismo espacio y desarrollan su vocación docente estén liderados, coordinados, orientados y dinamizados por ciertas personalidades destacadas dentro de la organización.

Según autores como Fuentes (2004, p. 104), Inglada, Sastre y Villarroya (2015, p. 137), los centros de enseñanza, concebidos bajo la fórmula cooperativa, son entendidos como entidades educativas que pretenden ofrecer una alternativa dentro de la bipolaridad del sistema educativo, centrando su proyecto en la innovación y la calidad del servicio ofertado. Estos centros abarcan un colectivo profesional aglutinado bajo las mismas necesidades laborales, creados bajo los principios de la cooperación, la democracia y el esfuerzo de un grupo de docentes.

Para Lorenzo (2004, p. 195), la implantación, estabilidad y desarrollo de este fenómeno social, emergente en los centros de naturaleza cooperativa, no solo van a depender del trabajo llevado a cabo por el líder, sino que van a influir de manera exponencial otros tipos de factores como pueden ser la predisposición de los trabajadores para realizar las tareas requeridas para alcanzar los objetivos, los recursos y medios disponibles, la colaboración e implicación de la dirección de la entidad, los mecanismos de resolución de problemas y las características del entorno que engloba a la entidad educativa.

La presente investigación se basa en el objetivo principal de conocer las percepciones docentes en torno al concepto de liderazgo en una cooperativa de enseñanza. De dicho objetivo se derivan otros más específicos, como son: reconocer al líder de una comunidad educativa, identificar valores como el compañerismo o el trabajo en cooperación, y por último, conocer el sistema relacional entre docentes de un mismo centro educativo de naturaleza cooperativa. 


\section{El liderazgo, un fenómeno emergente en los centros de enseñanza cooperativos}

El liderazgo es entendido por los expertos como una facultad de las personas, que poseen unas características individuales y peculiares que propician el seguimiento y cambios actitudinales del grupo que le rodea, con la finalidad de conseguir que el grupo ofrezca el máximo rendimiento en cada una de las acciones propuestas. Los líderes son capaces de transformar y concebir cada problema en el camino como un nuevo reto y oportunidad para marcar la diferencia con otros grupos de trabajo (Becerra y Sánchez, 2011, p. 64).

Para Castro (2006, p. 95), uno de los pilares que sustenta el liderazgo es su gran vínculo social, generado entre las personas que forman parte de un equipo de trabajo, creado para alcanzar unos fines, este hecho incide en la persecución del camino hacia el éxito. Además, el liderazgo es considerado un atributo de la personalidad, un poder influencial que estimula, genera confianza, motivación, cooperación y colaboración de las personas implicadas en el proceso de elaboración de determinadas acciones.

Toda organización de personas enfocada en la eficacia, como son los centros educativos cooperativos, deben tener presente el desarrollo del liderazgo entre sus miembros para originar distintos procesos de estimulación del rendimiento e implicación de todo el colectivo (Zamora y Poriet, 2006, p.65).

No obstante, uno de los aspectos más importantes a tener en cuenta en este fenómeno social emergente es la generación de ambientes y entornos positivos, que generen un clima óptimo para el desarrollo de cada una de las labores dentro del grupo. Por tanto, se debe evitar en cualquier situación el empleo de amenazas, chantajes o alzamientos de voz para obtener mayor autoridad frente al colectivo, puesto que se rompería todo nexo y clima afectivo producido y perjudicaría directamente al rendimiento (Castro, Miquilena y Peley, 2006, p. 89).

Los centros educativos deben creer en la implantación del liderazgo y confiar en las políticas y principios para alcanzar la máxima eficacia laboral de las personas que integran, de manera cooperativa, una organización (Gil, Alcover, Rico y Sánchez, 2011, p. 45).

El liderazgo se sustenta en la comunicación y en la voluntariedad. Gran parte del trabajo desarrollado por líderes se basa en procesos comunicativos constantes para lograr la máxima implicación y dedicación en las tareas a realizar por cada miembro de la entidad. No se debe olvidar que el forzar alguien a efectuar una acción con la que no está de acuerdo afecta al rendimiento y al clima laboral (Gutiérrez, Gracia y Quiñonez, 2016, p. 276). 


\subsection{El líder, una personalidad destacada en las instituciones escolares}

La tendencia actual del liderazgo se centra en la coexistencia de diversos perfiles de personas destacadas sobre los demás por sus cualidades y destrezas significativas que los diferencian del resto de docentes que integran el claustro. Se ha pasado de la concepción de un único líder a la teoría de la eficacia, mediante la distribución del poder influencial y dinamizador que pueden ejercer varias personalidades en el reparto y desarrollo de cada una de las acciones (Lorenzo, 2004, p. 196; Marín, 2004, p. 62; Pareja, 2009, p. 146; Pont, Nusche y Hopkins, 2008, p. 73).

Spillane, Camburn y Pareja (2009, p. 92) presentan tres modalidades de liderazgo distribuido en función del trabajo colaborativo llevado a cabo por docentes que ejercen de líderes.

- Liderazgo colaborativo: se produce cuando los líderes realizan la misma tarea de manera conjunta, en el mismo espacio.

- Liderazgo colectivo: se establece cuando los líderes llevan a cabo tareas totalmente distintas en entornos diferentes, pero contribuyen a la consecución de los objetivos.

- Liderazgo coordinado: se encuentra cuando los líderes desempeñan tareas diferentes según una coordinación entre ellos.

Autores como Rodríguez y Mármol (2009, p. 194), estudian el liderazgo formativo como una expansión del liderazgo, desarrollado en la educación. Este busca el compromiso, responsabilidad y formación continua de los integrantes del centro escolar para fomentar el desarrollo de la calidad en la educación. Para Cuevas, Díaz e Hidalgo (2008, p. 16), por un lado, el trabajo de los líderes se centra en movilizar, animar, motivar y dinamizar a las personas que componen el grupo, aumentando la autoestima e implicación. Por otro lado, se centra en observar, analizar, otorgar consejos y retroalimentaciones para orientar y guiar todo el proceso hacia la obtención del producto deseado, la calidad, tanto a nivel organizacional como en los procesos de enseñanza y aprendizaje.

Los líderes destacan por su constancia, trabajo, influencia, iniciativa, asertividad, empatía, comunicación, tolerancia, seguridad, reflexión y decisión, una serie de valores y cualidades que los diferencian del resto.

Para conseguir sus propósitos en el centro cooperativo, los líderes deben implantar un sistema democrático, basado en el respeto, donde se tengan en cuenta los intereses, necesidades e inquietudes de cada uno de los miembros (Furguerle y Vitorá, 2016, p. 213). 
Además, uno de los factores clave para la obtención de un máximo rendimiento y eficacia es que el personal docente se sienta que son las piezas más importantes de la cadena de producción. Para ello, los líderes tienen que centrar todos sus esfuerzos dinamizadores en obtener tal fin (Gil et al., 2011, p. 41).

Un aspecto esencial en el liderazgo es el principio de reciprocidad. Tanto los líderes como los seguidores o compañeros de estos deben trabajar en la misma medida, poner todo su empeño, dedicación, colaboración, sacrificio, entusiasmo en la realización de los ejercicios cotidianos (Becerra y Sánchez, 2011, p. 65; Guillen, 2006, p. 122).

En definitiva, la misión de los líderes educativos se centra en ejercer de coaches, guías, orientadores, coordinadores de las personas y gestores de los recursos para lograr la máxima productividad y reducir, en la medida de sus posibilidades, los momentos de tensión, frustración y nerviosismo para generar y potenciar entornos de seguridad y confianza que repercutirá positivamente en la realización de las tareas (Morales, 2012, p. 51; Pittinsky, 2009, p. 83; Zamora y Poriet, 2006, p. 65).

\subsection{Componentes esenciales del liderazgo}

Para lograr la máxima eficacia del liderazgo hay que tener en cuenta que este contiene un fuerte componente ético y, por tanto, necesita, según Goleman (2014, p. 35-51), disponer de inteligencia emocional, la cual se encuentra sustentada por los siguientes componentes:

- Autoconciencia: supone el conocimiento de uno mismo, conocer las emociones e impulsos, las potencialidades, limitaciones y la confianza para desempeñar determinadas funciones.

- Autogestión: se centra en la meditación, reflexión y control de los impulsos, sentimientos, motivaciones a la hora de realizar diversas tareas.

- Empatía: se basa en el respeto de los ideales y las creencias de las personas que nos rodean. Hay que saber situarse en el lugar de estas personas para comprender, en muchas ocasiones, su forma de pensar y actuar, sin criticar ni discriminar sus posturas planteadas o llevadas a cabo en la entidad.

- Social: hoy en día nadie se encuentra aislado de los demás, siempre hay alguien que establece un contacto o relación con otra persona. El líder debe fomentar las relaciones sociales dentro de la organización, debe crear vínculos y nexos de unión que promuevan el sentimiento de cohesión y lucha común por recorrer el camino hacia la meta de manera eficaz. 


\section{Metodología}

\subsection{Tipo de estudio}

El estudio que hemos desarrollado es de tipo descriptivo y correlacional, para ello se ha aplicado un análisis de datos desde un enfoque cuantitativo (Colás y Buendía, 1998, p. 63).

\subsection{Sujetos}

Para la investigación, hemos seleccionado a toda la población de docentes que conforman las cooperativas de enseñanza de la Ciudad Autónoma de Ceuta, esta se compone por tres centros educativos: Beatriz de Silva, Severo Ochoa y San Daniel. En todos ellos se imparten las etapas de Educación Infantil, Educación Primaria y Educación Secundaria.

La elección de la población es intencional, de tal forma que el criterio de selección de sujetos que se estableció para la investigación fue que los profesionales impartiesen docencia en el momento del estudio. La recopilación de datos se desarrolló durante el mes de septiembre de 2016 durante la celebración de los distintos claustros.

La población está formada por 60 sujetos, de los cuales se da una prevalencia de mujeres $(75 \%)$ que de hombres $(25 \%)$. El personal docente que imparte clases en tales cooperativas de enseñanza están concentrados en edades superiores a los 50 años $(46,7 \%)$, seguidos de los profesores que tienen entre 31 y 40 años (25\%), posteriormente, los que tienen entre 41 y 50 años $(23,3 \%)$ y finalmente, la franja menos numerosa es la que comprende las edades entre 20 y 30 años (5\%).

En relación con los años que llevan impartiendo docencia en las cooperativas de enseñanza, se concentran entre 11 y 20 años (50\%), seguidos de los que llevan más de 30 años $(21,7 \%)$, entre 0 y 10 años $(16,7 \%)$ y finalmente, los que llevan entre 21 y 30 años $(11,7 \%)$.

La titulación del personal docente es principalmente la diplomatura $(76,7 \%)$, seguida muy de lejos con titulaciones, tales como la licenciatura (15\%), grado $(6,7 \%)$ y doctorado $(1,7 \%)$. En cuanto al hecho que motivó su incorporación a las cooperativas de enseñanza, la mayoría lo hizo por vocación docente $(48,3 \%)$, seguido del hecho de acceder a un puesto de trabajo (35\%), tener plaza familiar $(8,3 \%)$ y por naturaleza empresarial $(8,3 \%)$. 


\subsection{Instrumento}

El instrumento que hemos aplicado para la investigación ha sido un cuestionario ad hoc, el cual fue creado específicamente para la obtención de datos de esta investigación en función de los objetivos especificados. Consta de 57 preguntas cerradas mediante escala Likert (1, Nada; 2, Poco; 3, Solo en parte; 4, Bastante). Estas cuestiones están distribuidas en cuatro ámbitos: Cooperativo (13), directivo (7), formación complementaria (7) y liderazgo (25), además de los datos sociodemográficos (sexo, edad, años de trabajo, grado de titulación académica y motivación laboral).

El instrumento ha pasado por el proceso de validación y fiabilidad. Para la validación hemos aplicado una validez de constructo por cinco doctores de diversas Universidades (Granada, Málaga y Sevilla), quienes recomendaron modificar aspectos gramaticales y eliminar ítems del ámbito liderazgo, aspectos que tuvimos en cuenta.

Además, aplicamos la fiabilidad mediante un coeficiente de consistencia interna de Alfa de Cronbach, a través de una prueba piloto aplicada cuatro meses antes de iniciar la investigación al grupo de 25 personas seleccionadas al azar de un centro educativo. En los resultados se obtiene una fiabilidad general del cuestionario superior a 0,85 de media, con unas valoraciones específicas en los diversos ámbitos, tal y como se refleja en la Tabla 1.

Tabla 1

Alfa de Cronbach

\begin{tabular}{|l|c|}
\hline \multicolumn{1}{|c|}{ Ámbito } & Alfa de Cronbach \\
\hline Cooperativo & 0.856 \\
\hline Directivo & 0.857 \\
\hline Formación Complementaria & 0.842 \\
\hline Liderazgo & 0.851 \\
\hline
\end{tabular}

Fuente: Elaboración propia (2017).

Para nuestra investigación, nos hemos centrado en las cuestiones sociodemográficas y el ámbito de liderazgo.

\subsection{Procedimiento}

El contacto inicial para la presentación de la investigación y solicitud de colaboración se realizó en los primeros días del mes de julio del año 2016 con los equipos directivos de las tres cooperativas de enseñanza, debido a que es el momento del año en el que mejor pueden atender, ya que no están en la vorágine de organización de final de curso. 
Presentado el proyecto y aceptado por parte de los tres equipos directivos, aprovechamos los diversos claustros celebrados a principios del curso 2016/2017. En ese momento, les indicamos las normas de cumplimentación del cuestionario para, posteriormente, mediante WhatsApp y enlace de correo electrónico pudieran acceder al mismo. No se les puso límite de tiempo para que lo pudieran rellenar con presiones, aunque el tiempo de finalización rondó entre los 40 y 50 minutos.

Durante su aplicación, el profesorado mostró mucha predisposición, de tal modo que no fue necesario resolver ningún tipo de dudas. Recopilada la información, se adaptaron los resultados obtenidos en Google Drive a SPSS, versión 20. En él se obtuvieron los datos y se procedió al análisis un mes después de presentación en los claustros.

\subsection{Variables y análisis de datos.}

Las variables del cuestionario son ordinales $(86,7 \%)$ y nominales $(13,3 \%)$. Las variables dependientes han sido sexo, edad, años de trabajo, grado de titulación académica y motivación laboral, mientras que las variables independientes son las 25 que conforman el ámbito liderazgo.

En el ámbito cooperativo tratamos de identificar los conocimientos del personal docente sobre cooperativas y su situación actual a nivel empresarial. En el ámbito directivo, analizamos las ventajas e inconvenientes de ser el órgano unipersonal del centro; en el ámbito de formación complementaria, observamos las necesidades, preferencias y frecuencia de formación de docentes; y en el ámbito liderazgo, identificamos los conocimientos sobre liderazgo, la opinión que tienen sobre sus líderes en dicho aspecto y el perfil líder que presentan los miembros que componen la cooperativa de enseñanza.

Se efectuó un análisis univariado y bivariado. Para el primero hemos obtenido de cada ítem las frecuencias y porcentajes, mientras que para el análisis bivariado hemos establecido diversas hipótesis y aplicado tablas de contingencia con el estadístico Chi-cuadrado, con un nivel de significancia inferior a 0,05 para rechazar la hipótesis de independencia, para tratar de identificar si existe o no independencia entre variables. En aquellos casos en los que no existía independencia, hemos aplicado la prueba Tau-b de Kendall, por ser ambas variables ordinales. Dada la extensión del artículo, hemos seleccionado aquellas variables que hemos considerado más significativas, las cuales tratan de dar respuesta al título planteado en el artículo. 


\section{Resultados y su análisis}

\subsection{Análisis univariado}

El personal docente que conforma las cooperativas de enseñanza conocen bastante el concepto de liderazgo (53\%), aunque existe un porcentaje bastante algo, más de un tercio, que conoce el concepto pero solo en parte. En contraprestación, son cifras muy bajas, alrededor del $20 \%$, los que desconocen o conocen poco el concepto de liderazgo (Figura 1).

Figura 1

Porcentaje de docentes con respecto al nivel de conocimiento del concepto de liderazgo, Ciudad Autónoma de Ceuta-España, 2017

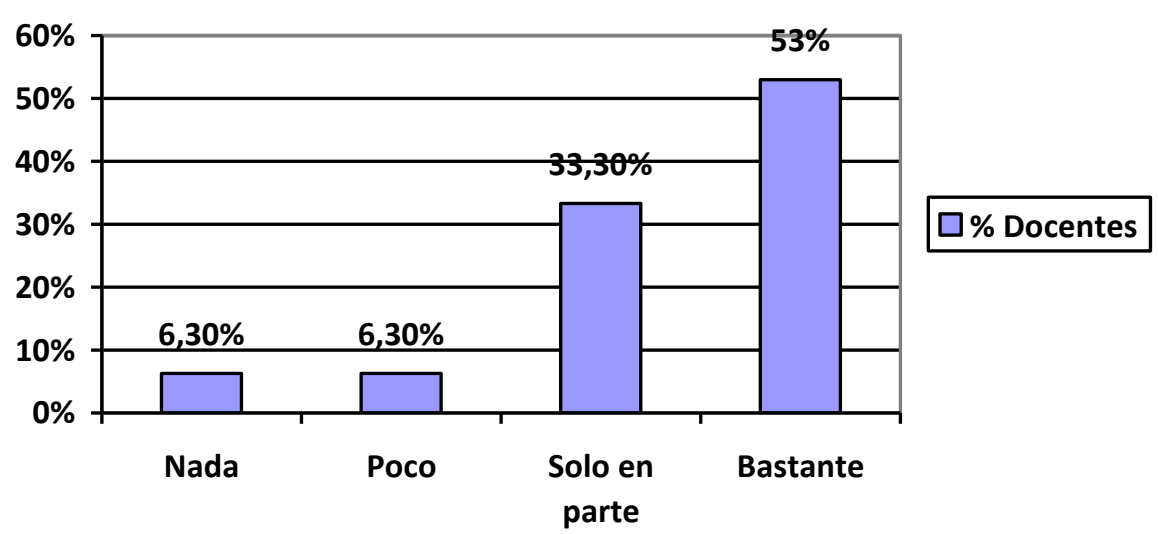

Fuente: Elaboración propia (2017).

A la hora de pensar si los directivos actuales de las cooperativas de enseñanza son auténticos líderes, la cuestión se encuentra más dividida, aunque en su mayoría las respuestas son afirmativas. Un 38,3\% de los encuestados cree que son auténticos líderes, pero solo en parte. Un 35\% los considera bastante líderes. Por otra parte, el 26,6\% piensa que sus directivos actuales son poco o nada auténticos líderes (Figura 2). 
Figura 2

Porcentaje de docentes con respecto a la concepción de los directivos como líderes de la entidad, Ciudad Autónoma de Ceuta-España, 2017

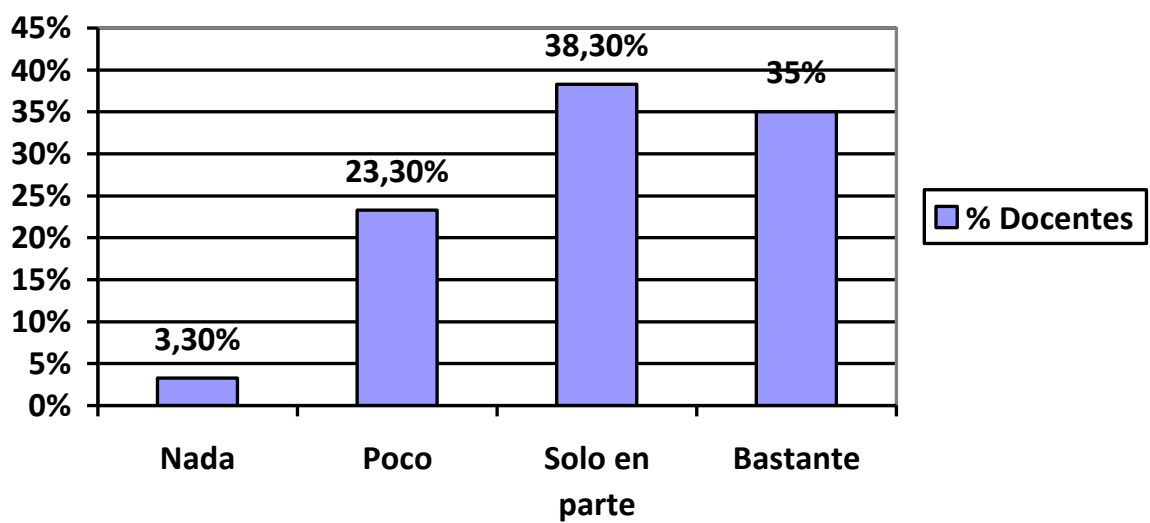

Fuente: Elaboración propia (2017).

La mayoría de las personas cooperativistas no han sido elegidas por sus colegas para un determinado cargo, concretamente el $45 \%$ de los socios. Un $33,3 \%$ ha sido elegido bastantes veces por sus compañeros para un determinado cargo, el resto de opciones son poco significativas (Figura 3).

Figura 3

Porcentaje de docentes con respecto al hecho de ser elegidos alguna vez para desempeñar un cargo directivo, Ciudad Autónoma de Ceuta-España, 2017

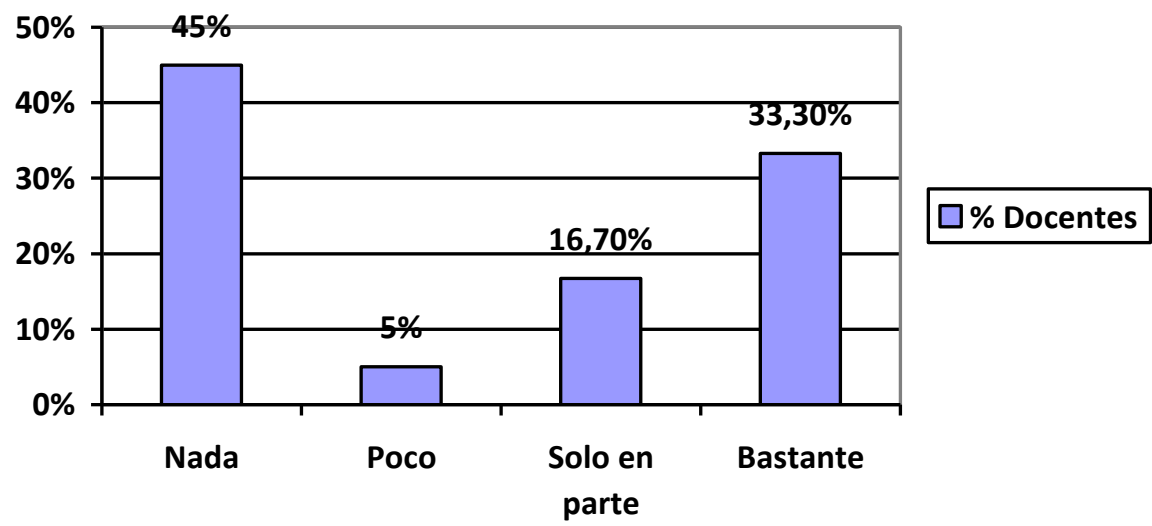

Fuente: Elaboración propia (2017).

Analizando los aspectos que marcan a un líder, en relación con si el personal docente organiza grupos de trabajo para desarrollar eficazmente tareas de diversa índole, nos encontramos con una cuestión bastante dividida en cuanto a opiniones. Cifras similares por 
encima del $30 \%$ aseguran que trabajan poco o nada en grupo. Un $25 \%$ lo hace solo en parte, lo que podemos interpretar como "en ocasiones". Por último, la cifra de "bastante", adquiere un escaso $3,3 \%$ (Figura 4).

Figura 4

Porcentaje de docentes con respecto al hecho de organizar grupos de trabajos para desarrollar tareas de diversa índole, Ciudad Autónoma de Ceuta-España, 2017

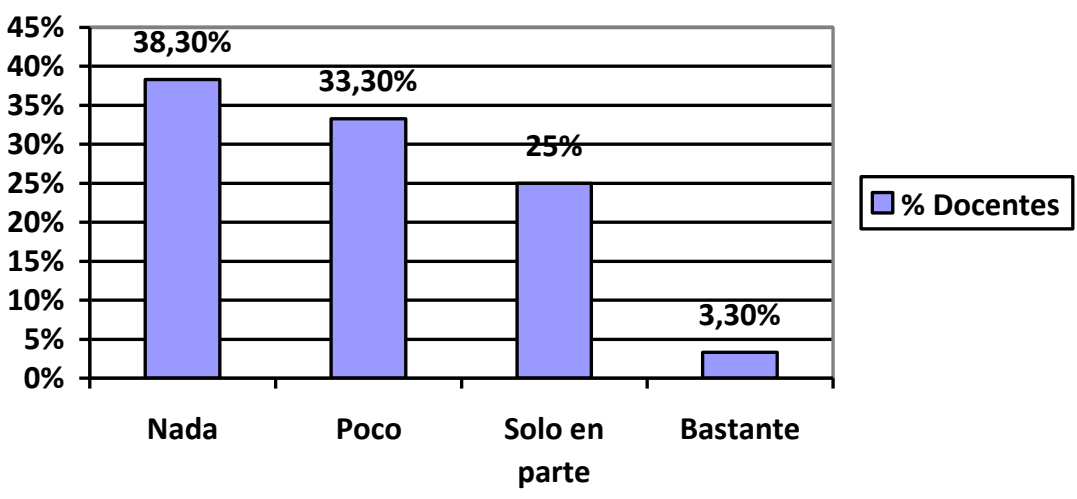

Fuente: Elaboración propia (2017).

Con respecto a si reparten las tareas a realizar entre docentes y colaboran en si para que el proceso de elaboración sea más eficiente, cuatro de cada diez aseguran que lo hacen en parte, seguidos por un $21,7 \%$ que lo hace bastante. Dos de cada diez personas no hacen nada, mientras que un $18,3 \%$ lo hacen poco (Figura 5).

Figura 5

Porcentaje de docentes con respecto al hecho de repartir tareas entre si y colaborar en el proceso de elaboración de estas, Ciudad Autónoma de Ceuta-España, 2017

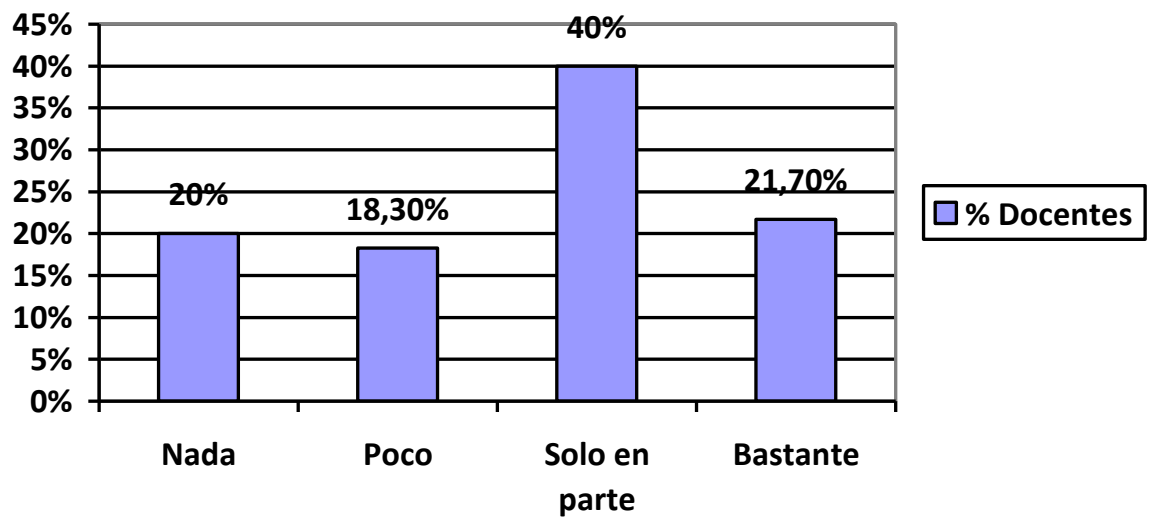

Fuente: Elaboración propia (2017). 
En cuanto a la defensa de ideales personales y respeto de la opinión del prójimo, sobre un determinado tema, la mayoría responde de manera positiva a la cuestión. Estas respuestas conforman un $78,3 \%$ del total, mientras que el resto lo hacen de manera negativa. Como observamos, una gran mayoría se muestra conforme con respetar las ideas de los compañeros, base fundamental de los movimientos cooperativos (Figura 6).

Figura 6

Porcentaje de docentes con respecto al hecho de defender las ideas sobre determinados temas respetando lo indicado por los compañeros, Ciudad Autónoma de Ceuta-España, 2017

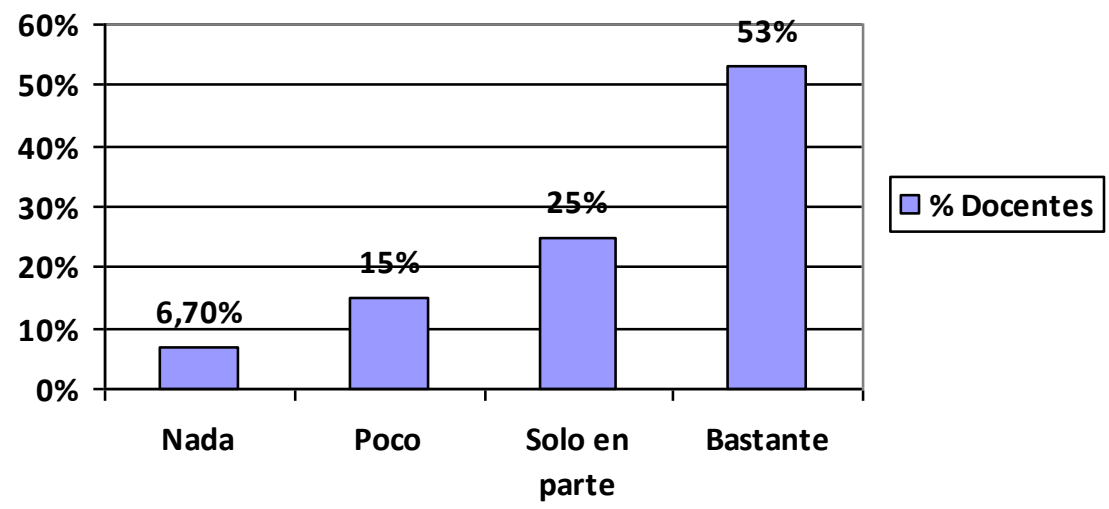

Fuente: Elaboración propia (2017).

En relación con si le piden consejo sobre alguna opinión relevante, un 43,3\% asevera que lo hace solo en parte, acompañado de un $21,7 \%$ que lo hace bastante. Un $21,7 \%$ de los entrevistados lo hacen poco, acompañados de un 13,3\% que no lo hace nada (Figura 7 ).

Figura 7

Porcentaje de docentes con respecto al hecho de pedir consejos u opinión sobre temas referentes al liderazgo, Ciudad Autónoma de Ceuta-España, 2017

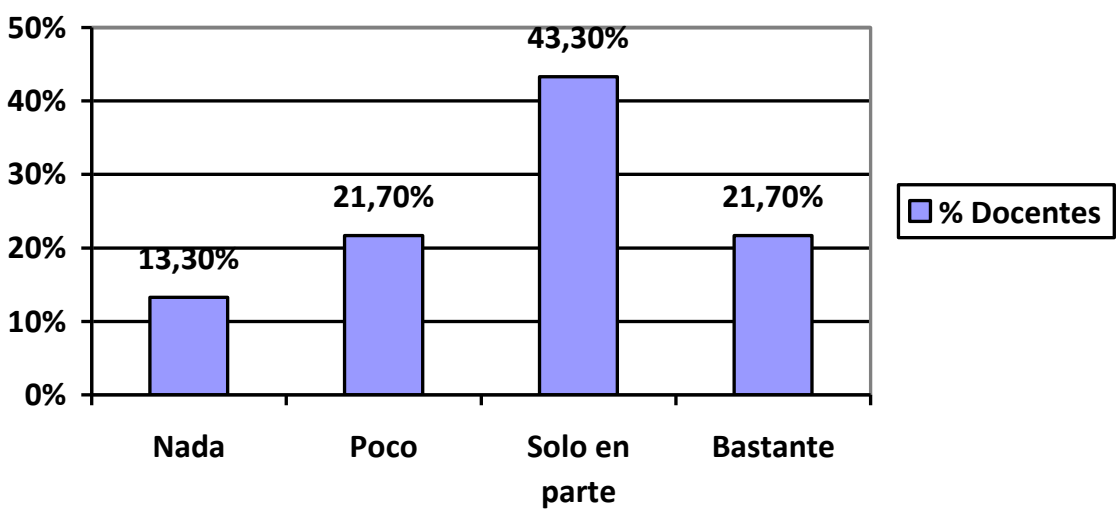

Fuente: Elaboración propia (2017). 
Con respecto a si es capaz de mantener la calma y confiar en sus compañeros en situaciones de estrés, la gran mayoría responde de manera positiva la cuestión, divididos entre un $50 \%$ que lo hace solo en parte y un $31,7 \%$ que lo hace bastante. Negativamente, responden que lo hacen poco un $15 \%$ y un 3,3\% que no lo hacen nada (Figura 8 ).

Figura 8

Porcentaje de docentes con respecto al hecho de mantener la calma y confiar en los compañeros en situaciones de estrés, Ciudad Autónoma de Ceuta-España, 2017

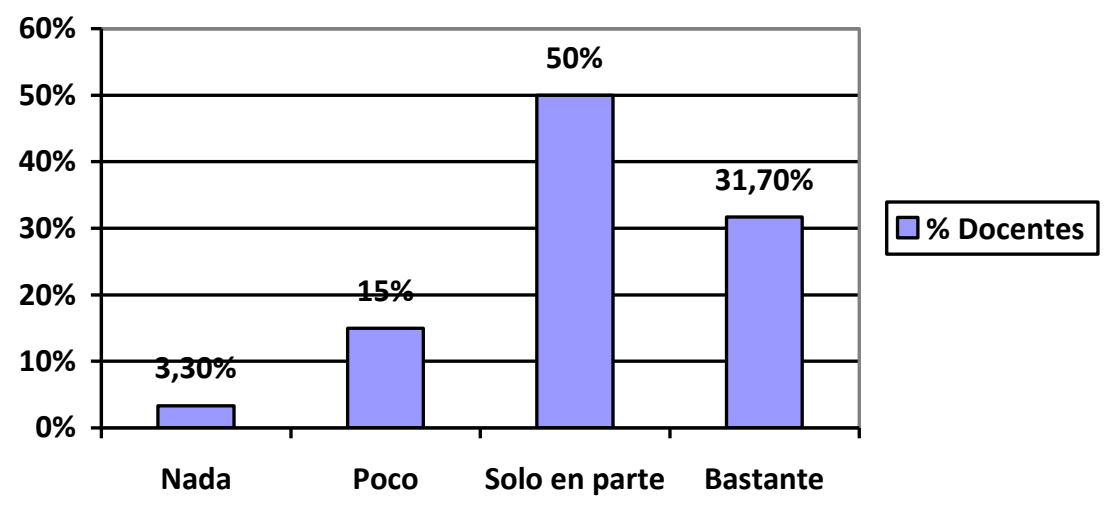

Fuente: Elaboración propia (2017).

Finalmente, en la cuestión sobre si piensa en sus compañeros/as y en el proceso que conlleva la consecución de una tarea o trabajo antes que en el resultado final, los resultados son parecidos a la cuestión anterior. Responden que lo hacen solo en parte, 5 de cada 10 entrevistados. Un $33,3 \%$ asegura que lo hace bastante, mientras que un $11,7 \%$ lo hace poco y un $5 \%$ no lo hace nada (Figura 9 ).

Figura 9

Porcentaje de docentes con respecto al pensamiento que tiene sobre los compañeros y el proceso que conlleva la consecución de una tarea antes que el producto final, Ciudad

Autónoma de Ceuta-España, 2017

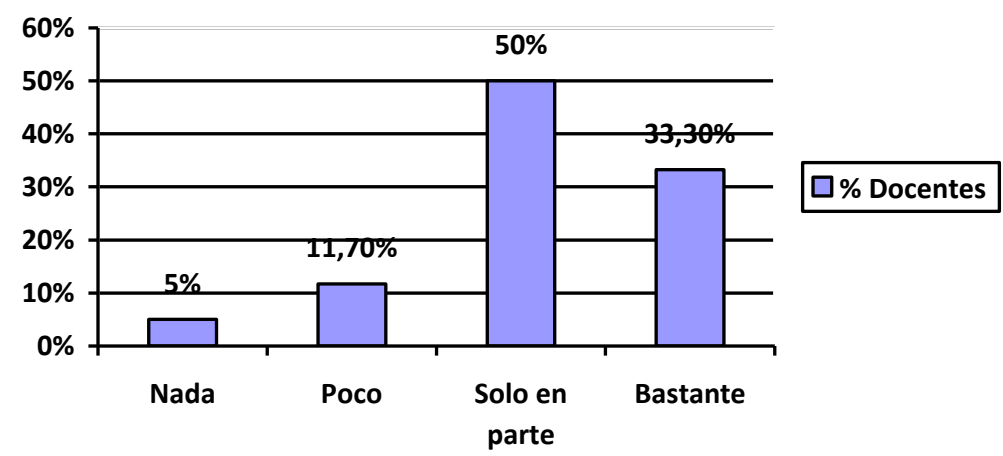

Fuente: Elaboración propia (2017). 


\subsection{Análisis bivariado}

En este apartado presentamos algunos de los resultados del análisis correlacional. En concreto, si existe relación o no en función del género del personal docente con el ámbito del liderazgo y el intervalo de edad (20-30, 31-40, 41-50 y más de 50 años).

\subsubsection{Género y liderazgo}

H1: "Hay relación entre hombre y mujeres en relación con el concepto que tienen las personas cooperativistas de liderazgo".

Resultado: Se acepta la hipótesis de independencia entre las dos variables. Se obtiene un p-valor en la prueba de Chi-cuadrado de Pearson de 0,682.

Interpretación: El hecho de ser hombre o mujer no influye en el concepto que las personas cooperativistas tienen sobre liderazgo $X^{2}(1 N=60)=0,682, p<.05$.

H2: "Hay relación entre hombres y mujeres con respecto a la concepción del directivo como líder".

Resultado: Se rechaza la hipótesis de independencia entre las dos variables. Se obtiene un p-valor en la prueba de Chi-cuadrado de Pearson de 0,009.

Interpretación: El hecho de ser hombre o mujer influye en el concepto que tienen sobre si sus directivos son auténticos líderes. En este caso, los hombres tienen una mejor valoración de sus directivos que las mujeres $X^{2}(1 N=60)=0,009, p<.05$.

Fuerza de asociación: Para ello, hemos establecido las dos variables de tipo nominal. Se ha obtenido un valor tanto en Phi como en $\mathrm{V}$ de Cramer de 0,432, por lo que se considera que la relación entre variables es media (valores entre 0,35 y 0,65).

H3: "Hay relación entre hombres y mujeres a la hora de ser elegidos por los compañeros para desempeñar funciones de equipo directivo".

Resultados: Se acepta la hipótesis de independencia entre las dos variables. Se obtiene un p-valor en la prueba de Chi-cuadrado de Pearson de 0,703.

Interpretación: El hecho de ser hombre o mujer no influye a la hora de elegir a un compañero para desempeñar la función de cargo directivo $X^{2}(1 \mathrm{~N}=60)=0,703, p<.05$.

H4: "Hay relación entre hombres y mujeres a la hora de organizar grupos de trabajo para desarrollar eficazmente tareas de diversa índole". 
Resultados: Se acepta la hipótesis de independencia entre las dos variables. Se obtiene un p-valor en la prueba de Chi-cuadrado de Pearson de 0,548.

Interpretación: El hecho de ser hombre o mujer no influye a la hora de organizar grupos de trabajo para desarrollar tareas de diversa índole $\mathrm{X}^{2}(1 \mathrm{~N}=60)=0,548, \mathrm{p}<.05$.

H5: "Hay relación entre hombres y mujeres a la hora de repartir las tareas a realizar entre docentes y colaborar entre si para que el proceso de elaboración sea más eficiente".

Resultados: Se acepta la hipótesis de independencia entre las dos variables. Se obtiene un p-valor en la prueba de Chi-cuadrado de Pearson de 0,800.

Interpretación: El hecho de ser hombre o mujer no influye a la hora de repartir las tareas entre docentes y colaborar entre si para que el proceso de elaboración sea más eficiente $\mathrm{X}^{2}(1 \mathrm{~N}=60)=0,800, \mathrm{p}<.05$.

H6: "Hay relación entre hombres y mujeres a la hora de defender sus ideas, con respeto del prójimo, sobre un determinado tema sin complejos".

Resultados: Se acepta la hipótesis de independencia entre las dos variables. Se obtiene un p-valor en la prueba de Chi-cuadrado de Pearson de 0,662.

Interpretación: El hecho de ser hombre o mujer no influye a la hora de defender sus ideas sobre un determinado tema $\mathrm{X}^{2}(1 \mathrm{~N}=60)=0,662, \mathrm{p}<.05$.

H7: "Hay relación entre hombres y mujeres a la hora de pedir consejo u opinión sobre algún tema relevante".

Resultados: Se acepta la hipótesis de independencia entre las dos variables. Se obtiene un p-valor en la prueba de Chi-cuadrado de Pearson de 0,388.

Interpretación: El hecho de ser hombre o mujer no incluye a la hora de pedir consejo u opinión sobre algún tema relevante $\mathrm{X}^{2}(1 \mathrm{~N}=60)=0,388, \mathrm{p}<.05$.

H8: "Hay relación entre hombres y mujeres a la hora de mantener la calma y confiar en sus compañeros en situaciones de estrés".

Resultados: Se acepta la hipótesis de independencia entre las dos variables. Se obtiene un p-valor en la prueba de Chi-cuadrado de Pearson de 0,317.

Interpretación: El hecho de ser hombre o mujer no influye a la hora de mantener la calma y confiar en los compañeros en situaciones de estrés $X^{2}(1 N=60)=0,317, p<.05$. 
H9: "Hay relación entre hombres y mujeres a la hora de pensar en los compañeros y en el proceso que conlleva la consecución de una tarea o trabajo antes que en el resultado final".

Resultados: Se acepta la hipótesis de independencia entre las dos variables. Se obtiene un p-valor en la prueba de Chi-cuadrado de Pearson de 0,657.

Interpretación: El hecho de ser hombre o mujer no influye a la hora de pensar en los compañeros y en el proceso que conlleva la consecución de una tarea o trabajo antes que el resultado final $\mathrm{X}^{2}(1 \mathrm{~N}=60)=0,657, \mathrm{p}<.05$.

\subsubsection{Intervalo de edad y liderazgo}

H10: "La edad determina el concepto que las personas cooperativistas tienen de liderazgo".

Resultado: Se acepta la hipótesis de independencia entre las dos variables. Se obtiene un p-valor en la prueba de Chi-cuadrado de Pearson de 0,619.

Interpretación: La edad no influye en el concepto que las personas cooperativistas tienen del liderazgo $\mathrm{X}^{2}(1 \mathrm{~N}=60)=0,619, \mathrm{p}<.05$.

H11: "La edad determina el concepto que las personas cooperativistas tienen acerca de si sus directivos son auténticos líderes".

Resultado: Se rechaza la hipótesis de independencia entre las dos variables. Se obtiene un p-valor en la prueba de Chi-cuadrado de Pearson de 0,200.

Interpretación: La edad no influye en el concepto que las personas cooperativistas tienen acerca de si sus directivos son auténticos líderes $X^{2}(1 N=60)=0,200, p<.05$.

H12: "La edad determina el hecho de ser elegidos por los compañeros para desempeñar funciones de equipo directivo".

Resultados: Se acepta la hipótesis de independencia entre las dos variables. Se obtiene un p-valor en la prueba de Chi-cuadrado de Pearson de 0,528.

Interpretación: La edad no influye a la hora de ser elegidos por los compañeros para desempeñar funciones de equipo directivo $\mathrm{X}^{2}(1 \mathrm{~N}=60)=0,528, \mathrm{p}<.05$.

H13: "La edad determina el hecho de organizar grupos de trabajo para desarrollar eficazmente tareas de diversa índole". 
Resultados: Se acepta la hipótesis de independencia entre las dos variables. Se obtiene un p-valor en la prueba de Chi-cuadrado de Pearson de 0,425.

Interpretación: La edad no influye a la hora de organizar los grupos de trabajo para desarrollar eficazmente tareas de diversa índole $\mathrm{X}^{2}(1 \mathrm{~N}=60)=0,425, \mathrm{p}<.05$.

H14: "La edad determina el hecho de repartir las tareas a realizar entre docentes y colaborar entre si para que el proceso de elaboración sea más eficiente".

Resultados: Se acepta la hipótesis de independencia entre las dos variables. Se obtiene un p-valor en la prueba de Chi-cuadrado de Pearson de 0,732.

Interpretación: La edad no influye a la hora de repartir las tareas a realizar entre docentes y colaborar entre si para que el proceso de elaboración sea más eficaz $\mathrm{X}^{2}(1 \mathrm{~N}=60)=0,732, \mathrm{p}<.05$.

H15: "La edad determina el hecho de defender sus ideas, con respeto del prójimo, sobre un determinado tema sin complejos".

Resultados: Se acepta la hipótesis de independencia entre las dos variables. Se obtiene un p-valor en la prueba de Chi-cuadrado de Pearson de 0,584.

Interpretación: La edad no influye a la hora de defender sus ideas, con respeto del prójimo, sobre un determinado tema sin complejos $\mathrm{X}^{2}(1 \mathrm{~N}=60)=0,584, \mathrm{p}<.05$.

H16: "La edad determina el hecho de pedir consejo u opinión sobre algún tema relevante".

Resultados: Se acepta la hipótesis de independencia entre las dos variables. Se obtiene un p-valor en la prueba de Chi-cuadrado de Pearson de 0,417.

Interpretación: La edad no influye a la hora de pedir consejo u opinión sobre algún tema relevante $\mathrm{X}^{2}(1 \mathrm{~N}=60)=0,417, \mathrm{p}<.05$.

H17: "La edad determina el hecho de mantener la calma y confiar en sus compañeros en situaciones de estrés".

Resultados: Se acepta la hipótesis de independencia entre las dos variables. Se obtiene un p-valor en la prueba de Chi-cuadrado de Pearson de 0,944.

Interpretación: La edad no influye a la hora de mantener la calma y confiar en sus compañeros en situaciones de estrés $X^{2}(1 \mathrm{~N}=60)=0,944, p<.05$. 
H18: "La edad determina el hecho de pensar en los compañeros y en el proceso que conlleva la consecución de una tarea o trabajo antes que en el resultado final".

Resultados: Se acepta la hipótesis de independencia entre las dos variables. Se obtiene un p-valor en la prueba de Chi-cuadrado de Pearson de 0,370.

Interpretación: La edad no influye a la hora de pensar en los compañeros y en el proceso que conlleva la consecución de una tarea o trabajo antes que en el resultado final $X^{2}(1 N=60)=0,370, p<.05$.

\section{Conclusiones}

Tras el análisis de los principales datos obtenidos, se han podido extraer las conclusiones más relevantes de esta investigación, las cuales manifiestan y reflejan la situación actual y el nivel de desarrollo del liderazgo en las cooperativas de enseñanza de Ceuta.

Se concreta que la mayoría de docentes conoce el concepto de liderazgo y todas las nociones que engloba. Además, el cuerpo docente manifiesta la consideración de que sus directivos son unos auténticos líderes, que gestionan, dirigen y coordinan la institución escolar de manera eficiente.

Con respecto a uno de los factores asociados al liderazgo como es el trabajo en equipo y reparto de tareas, se ha determinado que estos profesionales de la educación sí llevan a cabo un reparto equitativo de las acciones a realizar, pero trabajan de manera individualizada, con lo cual propician escasos procesos de interacción.

Otro de los puntos clave del liderazgo es el desarrollo de un ambiente propicio para el trabajo y la comunicación mediante un clima afectivo de relaciones personales. En este caso, la gran mayoría expone sus ideales y creencias sin ningún tipo de problema ante los miembros que componen el claustro, respetando las opiniones de los demás y siempre pensando en el bienestar de los compañeros de profesión antes que en los resultados finales.

En toda entidad educativa compuesta de una comunidad integrada por distintos colectivos, como docentes, discentes y familias de estos, siempre acontecen situaciones que provocan distracciones o descenso del rendimiento, como consecuencia de problemas que inciden, de manera directa o indirecta, en las labores cotidianas. No obstante, con base en los resultados obtenidos, se ha determinado que la amplia mayoría de docentes es capaz de 
mantener la calma en situaciones de estrés, lo que favorece exponencialmente el desarrollo del liderazgo.

No existen conexiones entre el género de los encuestados y el liderazgo, es decir, el género no influye en los elementos estudiados del liderazgo, excepto en la opinión que tienen tanto hombres como mujeres de sus directivos en cuanto a si son auténticos líderes. La relación existente entre ambas variables es media, por lo que se considera que los hombres presentan una mejor opinión de sus líderes que las mujeres, a pesar de que estas son las que mayoritariamente están presentes en las cooperativas de enseñanza. Lo mismo ocurre en relación con las conexiones existentes entre la edad de las personas cooperativistas y el liderazgo, en el que podemos afirmar que la edad no es un factor influyente en la opinión de este personal docente acerca del fenómeno estudiado.

El liderazgo que se pretende en toda organización social debe centrarse en el fomento del trabajo, esfuerzo, dedicación y compromiso de los miembros que la componen, con el fin de favorecer la consecución de los objetivos fijados. Los líderes, por tanto, tienen que enfocar sus acciones para gestionar y dinamizar a los trabajadores e influir en ellos para conseguir que todos caminen hacia el mismo sentido mediante reflexiones periódicas y la creación de vínculos afectivos, además de tener en cuenta las necesidades de cada uno de ellos y el conocimiento de sus puntos fuertes para que el reparto de tareas del grupo de trabajo sea más efectivo.

Para finalizar, la figura del líder debe presentarse como un ejemplo a seguir, todo un referente, una persona cercana, amable, con iniciativa, innovadora y dispuesta a ayudar a cualquier trabajador a desarrollar su cometido. La existencia de un liderazgo eficaz va a depender de la correcta conjugación de los diversos elementos que inciden en este fenómeno, tales como: la predisposición de los trabajadores, los recursos disponibles, las metas propuestas y los problemas que engloban todo el entorno de la entidad. El líder, en la educación, no debe solo incidir en quienes trabajan como docentes, sino en todos los miembros que componen la comunidad educativa. Es decir, en los alumnos y en el colectivo de padres, con el fin de mejorar la calidad de los procesos de enseñanza-aprendizaje y fomentar un buen clima de relaciones sociales.

\section{Referencias}

Becerra, Marlene y Sánchez, Ludy. (2011). El liderazgo en las organizaciones inteligentes. Revista científica digital del centro de investigación y estudios gerenciales, 1(4), 61-71. Recuperado de http://revista.grupocieg.org/publicacion/revista-cieg-n-4-mayo-2011/ 
Castro Solano, Alejandro. (2006). Teorías implícitas del liderazgo, contexto y capacidad de conducción. Anales de psicología, 22(1), 89-97. Recuperado de http://www.um.es/analesps/v22/v22 1/12-22 1.pdf

Castro, Elizabeth, Miquilena, Hermelinda y Peley, Rosario. (2006). Las nuevas tendencias del liderazgo: hacia una nueva visión de las organizaciones educativas. Omnia, 12(1), 83-96. Recuperado de http://www.produccioncientifica.luz.edu.ve/index.php/omnia/issue/view/864

Colás Bravo, María del Pilar, y Buendía Eisman, Leonor. (1998). Investigación Educativa. Sevilla: Ediciones Alfar.

Cuevas López, Mercedes, Díaz Rosas, Francisco e Hidalgo Hernández, Verónica. (2008). Liderazgo de los directores y calidad de la educación. Un estudio del perfil de los directivos en un contexto pluricultural. Profesorado. Revista de currículum y formación del profesorado, 12(2), 1-19. Recuperado de http://www.ugr.es/ recfpro/Rev122.html

Fuentes Viñas, Arturo. (2004). Las cooperativas de enseñanza: (un estudio de las cooperativas de trabajo asociado). Ceuta: Universidad Nacional de Educación a Distancia, Centro Asociado de Ceuta.

Furguerle Rangel, Johel y Vitorá, María. (2016). Liderazgo en los directivos de educación primaria. Telos: Revista de Estudios Interdisciplinarios en Ciencias Sociales, 18(2), 208-227. Recuperado de http://www.redalyc.org/pdf/993/99345727004.pdf

Gil, Francisco, Alcover, Carlos María, Rico, Ramón y Sánchez Manzanares, Miriam. (2011). Nuevas formas de liderazgo en equipos de trabajo. Papeles del psicólogo, 32(1), 3847. Recuperado de http://www.papelesdelpsicologo.es/contenido?num=1140

Goleman, Daniel. (2014). Liderazgo. El poder de la inteligencia emocional. Barcelona: Ediciones B.

Guillén Parra, Manuel. (2006). Ética en las organizaciones: construyendo confianza. Madrid: Pearson Prentice Hall.

Gutiérrez Vallejo, Ernesto, Gracia Chancay, José María y Quiñonez Mosquera, Jefferson. (2016). Liderazgo y gerencia de proyectos educativos y sociales. Dominio de las Ciencias, 2(4), 274-283. doi: https://doi.org/10.23857/pocaip

Inglada Galiana, Elena, Sastre Centeno, José Manuel y Villarroya Lequericaonandia, Begoña. (2015). El cooperativismo en la educación. REVESCO: Revista de estudios cooperativos, (118), 122-147. Recuperado de http://revistas.ucm.es/index.php/REVE/issue/view/2752/showToc

Lorenzo Delgado, Manuel. (2004). La función de liderazgo de la dirección escolar: una competencia transversal. Enseñanza, 22, 193-211. Recuperado de https://gredos.usal.es/ispui/bitstream/10366/70773/1/La funcion de liderazgo de la d ireccion.pdf 
Marín, José. (2004). La dirección, el liderazgo y la supervisión escolar. Caracas: Fondo Editorial Ipasme.

Morales Domínguez, José Francisco. (2012). Visión positiva del liderazgo y sus fortalezas. Madrid: Lección magistral UNED.

Pareja de la Reguera, José Antonio. (2009). Liderazgo y conflicto en las organizaciones educativas. Educación y educadores, 12(1), 137-152. Recuperado de http://www.scielo.org.co/pdf/eded/v12n1/v12n1a10.pdf

Pittinsky, Todd. (2009). Crossing the divide: Intergroup leadership in a world of difference. Boston: Harvard Business Press.

Pont, Beatriz, Nusche, Deborah y Hopkins, David. (2008). Improving School Leadership, Volume 2: Case Studies in System Leadership. Paris: Organisation for Economic Cooperation and Development.

Rodríguez Akle, Álvaro y Mármol Daza, Marta. (2009). Liderazgo formativo y desarrollo sostenible de la calidad de la educación. Investigación y desarrollo, 17(1), 192-207. Recuperado de http://rcientificas.uninorte.edu.co/index.php/investigacion/issue/view/51

Spillane, James, Camburn, Eric y Pareja, Amber. (2009). School principals at work: a distributed perspective. En Kenneth Leithwood, Blair Mascall y Tiiu. Strauss (Eds.), Distributed leadership according to the evidence (pp. 87-110). London: Routledge.

Zamora, Ángel y Poriet, Yenitza. (2006). Papel de los Líderes y Nuevas Tendencias del Liderazgo en el Siglo XXI. Revista Faces, 17(1), 61-73. Recuperado de http://servicio.bc.uc.edu.ve/faces/revista/vol27n1/art\%205.pdf 